TAIWANESE JOURNAL OF MATHEMATICS

Vol. 19, No. 1, pp. 159-174, February 2015

DOI: $10.11650 /$ tjm.19.2015.4068

This paper is available online at http://journal.taiwanmathsoc.org.tw

\title{
BURES DISTANCE FOR $\alpha$-COMPLETELY POSITIVE MAPS AND TRANSITION PROBABILITY BETWEEN $P$-FUNCTIONALS
}

\author{
Jaeseong Heo
}

\begin{abstract}
In this paper we discuss the Bures distance between $\alpha$-CP maps on a $C^{*}$-algebra and the transition probability between $P$-functionals on a *-algebra. We first review the notion of $\alpha$-CP maps and the representation theorem associated to $\alpha$-CP maps. Using the Krein space representation and the set of intertwiners between Krein space representations, we study the Bures distance between $\alpha$-CP maps. We prove that the transition probability between $P$-functionals can be estimated by some functionals using $J$-representations on Krein spaces.
\end{abstract}

\section{INTRODUCTION}

For two normal states $\mu, \nu$ on a von Neumann algebra $\mathcal{M}$, the Bures distance between $\mu$ and $\nu$ is defined as $\beta(\mu, \nu)=\inf \left\|x_{\mu}-y_{\nu}\right\|$ where the infimum is taken over all vectors $x_{\mu}$ and $y_{\nu}$ representing $\mu$ and $\nu$, respectively, in some common normal representation Hilbert space. In [6], Bures showed that $\beta:(\mu, \nu) \mapsto \beta(\mu, \nu)$ is a metric on the set of normal states of $\mathcal{M}$. This metric is a quantum generalization of the Fisher information metric in the quantum information and is identical to the FubiniStudy metric. Kretschmann, Schlingemann and Werner [16] extended the notion to completely positive linear maps from a $C^{*}$-algebra into $\mathcal{B}(\mathcal{H})$ using the Stinespring's dilation. Recently, Bhat and Sumesh [5] generalized this notion to completely positive linear maps between arbitrary $C^{*}$-algebras via Hilbert $C^{*}$-module language. They proved that the Bures distance may not be a metric when the range algebra is a general $C^{*}$-algebra and gave some examples with explicit computations of the Bures distance.

In quantum physics, the value $|\langle x, y\rangle|^{2}$ associated to unit vectors $x, y$ in a Hilbert space is interpreted as a transition probability between the corresponding states. Uhlmann

Received November 20, 2013, accepted May 13, 2014.

Communicated by Yuh-Jia Lee.

2010 Mathematics Subject Classification: Primary 46L89, 81P16; Secondary 46N50, 47L60.

Key words and phrases: $\alpha$-Completely positive map, Krein space, $J$-representation, Bures distance, Transition probability, $P$-functional.

This research was supported by Basic Science Research Program through the National Research Foundation of Korea(NRF) funded by the Ministry of Science, ICT and future Planning (2013R1A2A2A01006537). 
[19] introduced the transition probability $P(\mu, \nu)$ between two states $\mu, \nu$ of a *algebra $\mathcal{A}$, which is defined as the supremum of $\left|\left\langle x_{\mu}, y_{\nu}\right\rangle\right|^{2}$ where the supremum is taken over all vectors $x_{\mu}, y_{\nu}$ representing $\mu$ and $\nu$ as vector states in some common *-representation of $\mathcal{A}$. The transition probability for normal states of a von Neumann algebra $\mathcal{M}$ is related to the Bures distance by the formula $P(\mu, \nu)=\left(1-\frac{1}{2} \beta(\mu, \nu)^{2}\right)^{2}$. In particular, if $\mu$ and $\nu$ are states on the matrix algebra $M_{n}$ with density matrices $\rho_{\mu}$ and $\rho_{\nu}$, respectively, then $D_{1 / 2}\left(\rho_{\mu} \mid \rho_{\nu}\right) \geq \beta(\mu, \nu)^{2}$ where $D_{t}(t \in(0,1])$ is the quantum Tsallis relative entropy [1].

The completely positive maps are used as mathematical models for quantum instruments and quantum probability and has many applications in quantum information theory. However, in some local quantum field theories, the locality is in conflict with positivity, which leads to the modification of the positivity. From the motivation in local quantum field theory, we introduced the notion of $\alpha$-completely positive $(\alpha-\mathrm{CP})$ maps between (locally) $C^{*}$-algebras, which generalizes $\alpha$-positivity [15] and $P$-functionals [17]. The $\alpha$-complete positivity was studied in several papers $[8,9,10,11,12,13]$.

The purpose of the present paper is to study the Bures distance and the transition probability for $\alpha$-completely positive maps $\phi_{1}$ and $\phi_{2}$ on a $C^{*}$-algebra. In Section 2, we will formally introduce the Bures distance between $\alpha$-CP maps using the KSGNS representation theorem [9] for $\alpha$-CP maps and study the set of intertwiners between KSGNS representations associated to $\alpha$-CP maps. In Section 3, we estimate the $\mathrm{Bu}-$ res distance between $\alpha$-CP maps using the set of intertwiners and prove the triangle inequality the Bures distance between $\alpha$-CP maps. In Section 4, we study the transition probability between $P$-functionals for a $*$-algebra $\mathcal{A}$ using $J$-representations on Krein spaces and prove that the transition probability between $P$-functionals can be estimated by functionals which are bounded by given $P$-functionals and invariant under $\alpha=2 P-\operatorname{id}_{\mathcal{A}}$.

\section{Preliminaries}

Let $\mathcal{H}$ be a Hilbert space with the inner product $\langle\cdot, \cdot\rangle$ and let $J$ be a (fundamental) symmetry on $\mathcal{H}$, i.e., $J=J^{*}=J^{-1} \in \mathcal{B}(\mathcal{H})$. We define a sesquilinear form on $\mathcal{H}$ by

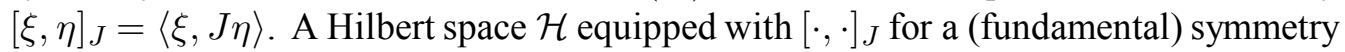
$J$ is called a Krein space or a $J$-space, denote by $(\mathcal{H}, J)$. For each $T \in \mathcal{B}(\mathcal{H})$ there exists an $T^{J} \in \mathcal{B}(\mathcal{H})$ such that

$$
[T \xi, \eta]_{J}=\left[\xi, T^{J} \eta\right]_{J}, \quad(\xi, \eta \in \mathcal{H}) .
$$

Indeed, we have that $[T \xi, \eta]_{J}=\langle T \xi, J \eta\rangle=\left\langle J \xi, J T^{*} J \eta\right\rangle=\left[\xi, J T^{*} J \eta\right]_{J}$, so that $T^{J}=J T^{*} J$ satisfies the equation (2.1). The operator $T^{J}$ is called a $J$-adjoint of $T$. Let $\mathcal{A}$ be a $C^{*}$-algebra and $\pi$ a representation of $\mathcal{A}$ on $\mathcal{H}$, where a representation means an algebra (not necessarily $*_{-}$) homomorphism. If there exists a symmetry $J$ on 
$\mathcal{H}$ such that

$$
[\pi(a) \xi, \eta]_{J}=\left[\xi, \pi\left(a^{*}\right) \eta\right]_{J} \quad \text { for any } a \in \mathcal{A} \text { and } \xi, \eta \in \mathcal{H},
$$

then $\pi$ is said to be a $J$-representation. It is easy to check that a representation $\pi$ is a $J$-representation if and only if $\pi\left(a^{*}\right)=\pi(a)^{J}$ for any $a \in \mathcal{A}$. We also see that $\|\pi(a)\|=\left\|\pi\left(a^{*}\right)\right\|$ for all $a \in \mathcal{A}$. Indeed, we have that

$$
\|\pi(a)\|^{2}=\left\|J \pi\left(a^{*}\right) J \pi(a)\right\| \leq\left\|\pi\left(a^{*}\right)\right\|\|\pi(a)\|,
$$

so that $\|\pi(a)\| \leq\left\|\pi\left(a^{*}\right)\right\|$. By symmetry, the converse inequality holds.

Let $\mathcal{B} \subset \mathcal{A}$ be unital $C^{*}$-algebras and $P$ a conditional expectation from $\mathcal{A}$ onto $\mathcal{B}$, that is, $P\left(1_{\mathcal{A}}\right)=1_{\mathcal{A}}, P\left(b_{1} a b_{2}\right)=b_{1} P(a) b_{2}$ and $P\left(a^{*}\right)=P(a)^{*}$ for any $a \in \mathcal{A}$ and $b_{1}, b_{2} \in \mathcal{B}$. A Hermitian linear functional $\rho$ on $\mathcal{A}$ is called a $P$-functional on $\mathcal{A}$ if the following conditions are satisfied:

(i) $\rho(P(a))=\rho(a)$ for any $a \in \mathcal{A}$,

(ii) $2 \rho\left(P(a)^{*} P(a)\right) \geq \rho\left(a^{*} a\right)$ for any $a \in \mathcal{A}$.

If $\alpha: \mathcal{A} \rightarrow \mathcal{A}$ is defined by $\alpha(a)=2 P(a)-a$, then it is easy to check that

$$
\rho\left(\alpha\left(a_{1}\right) \alpha\left(a_{2}\right)\right)=\rho\left(a_{1} a_{2}\right) \quad \text { and } \quad \rho\left(\alpha(a)^{*} a\right) \geq 0
$$

for any $a_{1}, a_{2}, a \in \mathcal{A}[4]$.

\section{1. $\alpha$-CP maps on $C^{*}$-algebras and Krein space representations}

Motivated by the $\alpha$-positivity [15] and $P$-functionals in [4, 14, 17], we introduced a notion of $\alpha$-completely positive maps as a generalization of completely positive maps [9]. Let $\phi: \mathcal{A} \rightarrow \mathcal{B}(\mathcal{H})$ be a Hermitian map. Then for any $n \geq 1$ and $a_{i j} \in \mathcal{A}$ $(1 \leq i, j \leq n)$, we have $\phi_{n}\left[\left(a_{i j}\right)^{*}\right]=\left[\phi\left(a_{j i}{ }^{*}\right)\right]=\left[\phi\left(a_{i j}\right)\right]^{*}$, so that $\phi_{n}$ is Hermitian for any $n \geq 1$.

Throughout this paper, $\mathcal{A}$ and $\mathcal{H}$ denote a unital $C^{*}$-algebra with unit $1_{\mathcal{A}}$ and a Hilbert space over $\mathbb{C}$, respectively, unless specified otherwise.

Definition 2.1. A Hermitian linear map $\phi$ of $\mathcal{A}$ into $\mathcal{B}(\mathcal{H})$ is called $\alpha$-completely positive (briefly, $\alpha-C P$ ) if there is a bounded Hermitian linear map $\alpha: \mathcal{A} \rightarrow \mathcal{A}$ such that

(i) $\alpha^{2}=\operatorname{id}_{\mathcal{A}}$ where $\operatorname{id}_{\mathcal{A}}$ is the identity map on $\mathcal{A}$,

(ii) $\alpha\left(1_{\mathcal{A}}\right)=1_{\mathcal{A}}$,

(iii) $\phi(a b)=\phi(\alpha(a) \alpha(b))$ for any $a, b \in \mathcal{A}$,

(iv) for any $n \geq 1, a_{1}, \cdots, a_{n} \in \mathcal{A}$ and $\xi_{1}, \cdots, \xi_{n} \in \mathcal{H}$,

$$
\sum_{i, j=1}^{n}\left\langle\phi\left(\alpha\left(a_{j}\right)^{*} a_{i}\right) \xi_{i}, \xi_{j}\right\rangle \geq 0
$$


(v) for each $a, a_{1}, \ldots, a_{n} \in \mathcal{A}$, there exists a constant $C(a) \geq 0$ such that

$$
\left(\phi\left(\alpha\left(a a_{j}\right)^{*} a a_{i}\right)\right) \leq C(a)\left(\phi\left(\alpha\left(a_{j}\right)^{*} a_{i}\right)\right)
$$

where a big parenthesis denotes an $n \times n$ matrix.

In case where $\alpha=\operatorname{id}_{\mathcal{A}}$ in Definition 2.1, the $\alpha$-complete positivity implies the complete positivity. Moreover, the equality $\phi(a b)=\phi(\alpha(a b))$ immediately follows from the condition (ii) in Definition 2.1 since $\phi(a b)=\phi\left(a b \cdot 1_{\mathcal{A}}\right)=\phi\left(\alpha(a b) \alpha\left(1_{\mathcal{A}}\right)\right)=$ $\phi(\alpha(a b))$.

See [9] for the example of an $\alpha$-CP map on the $2 \times 2$-matrix algebra which is not completely positive. It is known that in massless quantum field theory the state space will be a space with indefinite metric. Motivated by this physical fact, many people extended the GNS construction to Krein spaces. More generally, Heo-HongJi in [9] provided such KSGNS representations on a Krein module for $\alpha$-completely positive maps on a $C^{*}$-algebra or a *-algebra. For the reader's convenience, we begin by sketching the Stinespring type construction associated with an $\alpha$-completely positive map which will be needed in this paper.

Theorem 2.2. [10]. If $\phi: \mathcal{A} \rightarrow \mathcal{B}(\mathcal{H})$ is an $\alpha-C P$ map, then there exist a Krein space $(\mathcal{K}, J)$, a unital J-representation $\pi$ of $\mathcal{A}$ on $\mathcal{K}$ and a bounded operator $V \in \mathcal{B}(\mathcal{H}, \mathcal{K})$ such that

(i) $\phi(a)=V^{*} \pi(a) V$, so that $\phi\left(a^{*}\right)=V^{*} \pi(a)^{J} V$,

(ii) $V^{*} \pi(a)^{*} \pi(b) V=V^{*} \pi\left(\alpha(a)^{*} b\right) V$,

(iii) the set $\{\pi(a) V \xi: a \in \mathcal{A}, \xi \in \mathcal{H}\}$ is total in $\mathcal{K}$.

We call the quadruple $(\mathcal{K}, J, \pi, V)$ in Theorem 2.2 the Krein quadruple associated with an $\alpha-C P$ map $\phi$ if it satisfies conditions (i) and (ii). In this case, we say that $(\mathcal{K}, J, \pi, V)$ dilates $\phi$.

Remark 2.3. Let $(\mathcal{K}, J, \pi, V)$ be the quadruple constructed in Theorem 2.2. We note that the equality $J V=V$ holds. If $\left(\mathcal{K}^{\prime}, J^{\prime}, \pi^{\prime}, V^{\prime}\right)$ is another quadruples satisfying properties (i), (ii) and (iii) in Theorem 2.2 and if the equality $J^{\prime} V^{\prime}=V^{\prime}$ holds, then there is a unitary operator $U$ in $\mathcal{B}\left(\mathcal{K}, \mathcal{K}^{\prime}\right)$ such that

$$
\pi^{\prime}(a)=U \pi(a) U^{*} \quad(a \in \mathcal{A}), \quad V^{\prime}=U V \quad \text { and } \quad J^{\prime}=U J U^{*} .
$$

See Theorem 4.6 and Remark 4.7 in [10] for the proof.

If a quadruple $\left(\mathcal{K}^{\prime}, J^{\prime}, \pi^{\prime}, V^{\prime}\right)$ satisfies properties (i), (ii) and (iii) in Theorem 2.2 and if the equality $J^{\prime} V^{\prime}=V^{\prime}$ holds, then it is called a minimal dilation associated with $\phi$. This minimal dilation is unique up to unitary equivalence. 


\subsection{Bures distance between $\alpha$-CP maps on $C^{*}$-algebras}

We denote by $\alpha-\operatorname{CP}(\mathcal{A}, \mathcal{H})$ the set of all $\alpha$-CP map $\phi$ of $\mathcal{A}$ into $\mathcal{B}(\mathcal{H})$. For any $\phi_{i}$ $(i=1,2)$ in $\alpha-\operatorname{CP}(\mathcal{A}, \mathcal{H})$, let $\left(\mathcal{K}_{i}, J_{i}, \pi_{i}, V_{i}\right)$ be a minimal Krein quadruple associated with $\phi_{i}$. Take $\mathcal{K}=\mathcal{K}_{1} \oplus \mathcal{K}_{2}, J=J_{1} \oplus J_{2}, \pi=\pi_{1} \oplus \pi_{2}, \widetilde{V}_{1}=V_{1} \oplus 0$ and $\widetilde{V}_{2}=0 \oplus V_{2}$. We easily see that

$$
\begin{aligned}
\phi_{i}(a) & =\widetilde{V}_{i}^{*} \pi(a) \widetilde{V}_{i}, \\
\widetilde{V}_{i}^{*} \pi\left(\alpha(a)^{*} b\right) \widetilde{V}_{i} & =\widetilde{V}_{i}^{*} \pi(a)^{*} \pi(b) \widetilde{V}_{i} .
\end{aligned}
$$

Thus, the quadruple $\left(\mathcal{K}, J, \pi, \widetilde{V}_{i}\right)$ dilates $\phi_{i}(i=1,2)$. In this case, we call the triple $(\mathcal{K}, J, \pi)$ the common representation for $\phi_{1}$ and $\phi_{2}$.

Let $(\mathcal{K}, J)$ be a Krein space and let $\pi: \mathcal{A} \rightarrow \mathcal{B}(\mathcal{K})$ be a $J$-representation. We denote by $S(\phi, \pi)$ the set of all bounded operators $V \in \mathcal{B}(\mathcal{H}, \mathcal{K})$ such that $(\mathcal{K}, J, \pi, V)$ dilates $\phi \in \alpha-\mathrm{CP}(\mathcal{A}, \mathcal{H})$. Here, we don't demand minimality for Krein quadruples.

Definition 2.4. (cf. [16]) Let $\phi_{i}(i=1,2)$ be any element of $\alpha-\mathrm{CP}(\mathcal{A}, \mathcal{H})$.

(i) The $\pi$-distance between $\phi_{1}$ and $\phi_{2}$ is defined as

$$
\beta_{\pi}\left(\phi_{1}, \phi_{2}\right)=\inf \left\{\left\|V_{1}-V_{2}\right\|: V_{i} \in S\left(\phi_{i}, \pi\right)\right\} .
$$

(ii) The Bures distance between $\phi_{1}$ and $\phi_{2}$ is the smallest $\pi$-distance

$$
\beta\left(\phi_{1}, \phi_{2}\right)=\inf _{\pi} \beta_{\pi}\left(\phi_{1}, \phi_{2}\right)
$$

where the infimum is taken over all representations $\left(\mathcal{K}, J, \pi, V_{i}\right)$ which dilates $\phi_{i}$.

\subsection{Intertwiners between Krein quadruples associated with $\alpha$-CP maps}

For each $i=1,2$, let $\left(\mathcal{K}_{i}, J_{i}, \pi_{i}, V_{i}\right)$ be a Krein quadruple associated with $\phi_{i}$ in $\alpha-\operatorname{CP}(\mathcal{A}, \mathcal{H})$. For two maps $\phi_{1}, \phi_{2}$ in $\alpha-\operatorname{CP}(\mathcal{A}, \mathcal{H})(i=1,2)$, we define the set

$$
\begin{aligned}
\mathcal{I}\left(\phi_{1}, \phi_{2}\right) & =\left\{V_{1}^{*} W V_{2}: W \in \mathcal{B}\left(\mathcal{K}_{2}, \mathcal{K}_{1}\right) \text { with }\|W\| \leq 1,\right. \\
W J_{2} & \left.=J_{1} W \text { and } W \pi_{2}(a)=\pi_{1}(a) W \forall a \in \mathcal{A}\right\} .
\end{aligned}
$$

If $\left(\widehat{\mathcal{K}}_{i}, \widehat{J}_{i}, \widehat{\pi}_{i}, \widehat{V}_{i}\right)$ is a minimal Krein quadruple associated with $\phi_{i}$, by uniqueness of a minimal Krein quadruple, there exists an isometry $U_{i}: \widehat{\mathcal{K}}_{i} \rightarrow \mathcal{K}_{i}$ such that

$$
U_{i} \widehat{V}_{i}=V_{i}, \quad U_{i} \widehat{J}_{i}=J_{i} U_{i}, \quad \pi_{i}(a)=U_{i} \widehat{\pi}_{i}(a) U_{i}^{*} \quad(a \in \mathcal{A}) .
$$

Since $U_{i} U_{i}^{*}$ is a projection onto the closed linear span of the set $\left\{\pi_{i}(a) V_{i} \xi: a \in \mathcal{A}, \xi \in\right.$ $\mathcal{H}\}$, we have that $U_{i} U_{i}^{*} V_{i}=V_{i}$. For any $W \in \mathcal{I}\left(\phi_{1}, \phi_{2}\right)$, we see that

$$
V_{1}^{*} W V_{2}=V_{1}^{*} U_{1} U_{1}^{*} W U_{2} U_{2}^{*} V_{2}=\widehat{V}_{1}^{*} U_{1}^{*} W U_{2} \widehat{V_{2}}=\widehat{V}_{1}^{*} \widehat{W} \widehat{V_{2}}
$$


where $\widehat{W}:=U_{1}^{*} W U_{2}$ is a contractive operator from $\widehat{\mathcal{K}_{2}}$ into $\widehat{\mathcal{K}_{1}}$. It follows from (2.3) that

$$
\widehat{W} \widehat{\pi_{2}}(a)=U_{1}^{*} W \pi_{2}(a) U_{2}=\widehat{\pi_{1}}(a) U_{1}^{*} W U_{2}=\widehat{\pi_{1}}(a) \widehat{W},
$$

and that

$$
\widehat{W} \widehat{J}_{2}=U_{1}^{*} W J_{2} U_{2}=\widehat{J}_{1} U_{1}^{*} W U_{2}=\widehat{J_{1}} \widehat{W}
$$

This implies that

$$
\mathcal{I}\left(\phi_{1}, \phi_{2}\right) \subseteq \widehat{\mathcal{I}}\left(\phi_{1}, \phi_{2}\right):=\left\{\widehat{V}_{1}^{*} \widehat{W} \widehat{V_{2}}: \widehat{W} \widehat{J}_{2}=\widehat{J_{1}} \widehat{W}, \widehat{W} \widehat{\pi_{2}}(a)=\widehat{\pi_{1}}(a) \widehat{W} \forall a \in \mathcal{A}\right\}
$$

where $\left(\widehat{\mathcal{K}}_{i}, \widehat{J}_{i}, \widehat{\pi}_{i}, \widehat{V}_{i}\right)$ is a minimal Krein quadruple associated to $\phi_{i}(i=1,2)$. By taking $W=U_{1} \widehat{W} U_{2}^{*}$, we similarly get the reverse inclusion.

For two $\alpha$-CP maps $\phi_{i}(i=1,2)$ and a $J$-representation $\pi$ of $\mathcal{A}$ on a Krein space $(\mathcal{K}, J)$, we define the sets

$$
\begin{aligned}
\mathcal{J}_{\pi}\left(\phi_{1}, \phi_{2}\right) & =\left\{V_{1}^{*} V_{2}: V_{i} \in S\left(\phi_{i}, \pi\right)\right\} \subset \mathcal{B}(\mathcal{H}) \\
\mathcal{J}\left(\phi_{1}, \phi_{2}\right) & =\bigcup_{\pi} \mathcal{J}_{\pi}\left(\phi_{1}, \phi_{2}\right)
\end{aligned}
$$

where the union is over all representations $\pi$ of $\mathcal{A}$, admitting a common Krein space dilation for $\phi_{1}$ and $\phi_{2}$.

Proposition 2.5. If $\phi_{i}: \mathcal{A} \rightarrow \mathcal{B}(\mathcal{H})$ is two $\alpha$-CP maps $(i=1,2)$, then

$$
\mathcal{I}\left(\phi_{1}, \phi_{2}\right)=\mathcal{J}\left(\phi_{1}, \phi_{2}\right) \text {. }
$$

Proof. Let $\left(\widehat{\mathcal{K}}_{i}, \widehat{J}_{i}, \widehat{\pi}_{i}, \widehat{V}_{i}\right)$ be a minimal Krein quadruple associated with $\phi_{i}$ and let $\widehat{W}: \widehat{\mathcal{K}_{2}} \rightarrow \widehat{\mathcal{K}_{1}}$ with $\|\widehat{W}\| \leq 1$ be such that

$$
\widehat{W} \widehat{J}_{2}=\widehat{J_{1}} \widehat{W}, \quad \text { and } \quad \widehat{W} \widehat{\pi_{2}}(a)=\widehat{\pi_{1}}(a) \widehat{W} \quad \text { for all } a \in \mathcal{A} \text {. }
$$

Define bounded linear operators $V_{i}: \mathcal{H} \rightarrow \widehat{\mathcal{K}_{1}} \oplus \widehat{\mathcal{K}_{2}}$ by

$$
V_{1}=\widehat{V_{1}} \oplus 0 \quad \text { and } \quad V_{2}=\widehat{W} \widehat{V_{2}} \oplus\left(1_{\widehat{\mathcal{K}}_{2}}-\widehat{W}^{*} \widehat{W}\right)^{1 / 2} \widehat{V}_{2}
$$

Let $a \in \mathcal{A}$. We easily see that $\phi_{1}(a)=V_{1}^{*}\left(\widehat{\pi_{1}}(a) \oplus \widehat{\pi_{2}}(a)\right) V_{1}$ and that

$$
V_{1}^{*}\left(\widehat{\pi_{1}}(a) \oplus \widehat{\pi_{2}}(a)\right)^{*}\left(\widehat{\pi_{1}}(b) \oplus \widehat{\pi_{2}}(b)\right) V_{1}=V_{1}^{*}\left(\widehat{\pi_{1}}\left(\alpha(a)^{*} b\right) \oplus \widehat{\pi_{2}}\left(\alpha(a)^{*} b\right)\right) V_{1} .
$$

We also have that

$$
\begin{aligned}
V_{2}^{*}\left(\widehat{\pi_{1}}(a) \oplus \widehat{\pi_{2}}(a)\right) V_{2} & ={\widehat{V_{2}}}^{*} \widehat{W}^{*} \widehat{\pi_{1}}(a) \widehat{W} \widehat{V_{2}}+{\widehat{V_{2}}}^{*}\left(1_{\widehat{\mathcal{K}_{2}}}-\widehat{W}^{*} \widehat{W}\right) \widehat{\pi_{2}}(a) \widehat{V_{2}} \\
& =\widehat{V}_{2}^{*} \widehat{\pi_{2}}(a) \widehat{V_{2}}=\phi_{2}(a)
\end{aligned}
$$


and that

$$
\begin{aligned}
V_{2}^{*} & \left(\widehat{\pi_{1}}(a) \oplus \widehat{\pi_{2}}(a)\right)^{*}\left(\widehat{\pi_{1}}(b) \oplus \widehat{\pi_{2}}(b)\right) V_{2} \\
& =\widehat{V}_{2}^{*} \widehat{W}^{*} \widehat{\pi_{1}}(a)^{*} \widehat{\pi_{1}}(b) \widehat{W} \widehat{V_{2}}+\widehat{V}_{2}^{*}\left(1_{\widehat{\mathcal{K}_{2}}}-\widehat{W}^{*} \widehat{W}\right) \widehat{\pi_{2}}(a)^{*} \widehat{\pi_{2}}(b) \widehat{V_{2}} \\
& =V_{2}^{*}\left(\widehat{\pi_{1}}\left(\alpha(a)^{*} b\right) \oplus \widehat{\pi_{2}}\left(\alpha(a)^{*} b\right)\right) V_{2} .
\end{aligned}
$$

Let $J=J_{1} \oplus J_{2}$ and $\pi=\widehat{\pi_{1}} \oplus \widehat{\pi_{2}}$. For any $a \in \mathcal{A}$, we have that

$$
\pi(a)^{J}=\left(J_{1} \oplus J_{2}\right)\left(\widehat{\pi_{1}}(a) \oplus \widehat{\pi_{2}}(a)\right)^{*}\left(J_{1} \oplus J_{2}\right)=\pi\left(a^{*}\right),
$$

so that $\pi=\widehat{\pi_{1}} \oplus \widehat{\pi_{2}}$ is a $J$-representation of $\mathcal{A}$ on $\left(\widehat{\mathcal{K}_{1}} \oplus \widehat{\mathcal{K}_{2}}, \widehat{J_{1}} \oplus \widehat{J_{2}}\right)$. Hence, the quadruple $\left(\widehat{\mathcal{K}_{1}} \oplus \widehat{K_{2}}, \widehat{J_{1}} \oplus \widehat{J_{2}}, \widehat{\pi_{1}} \oplus \widehat{\pi_{2}}, V_{i}\right)$ dilates each $\phi_{i}(i=1,2)$. Moreover, we have that

$$
V_{1}^{*} V_{2}=\left(\widehat{V_{1}} \oplus 0\right)^{*}\left(\widehat{W} \widehat{V_{2}} \oplus\left(1_{\widehat{\mathcal{K}}_{2}}-\widehat{W}^{*} \widehat{W}\right)^{1 / 2} \widehat{V_{2}}\right)=\widehat{V}_{1}^{*} \widehat{W} \widehat{V_{2}},
$$

which implies that $\mathcal{J}\left(\phi_{1}, \phi_{2}\right) \neq \emptyset$ and $\mathcal{I}\left(\phi_{1}, \phi_{2}\right) \subseteq \mathcal{J}\left(\phi_{1}, \phi_{2}\right)$.

For the reverse inclusion, let $\pi$ be any common $J$-representation of $\mathcal{A}$ on $(\mathcal{K}, J)$ for $\phi_{1}$ and $\phi_{2}$ and let $V_{1}^{*} V_{2} \in \mathcal{J}_{\pi}\left(\phi_{1}, \phi_{2}\right)$. We denote by $\mathcal{K}_{2}$ the closed linear span of $\left\{\pi(a) V_{2} \xi: a \in \mathcal{A}, \xi \in \mathcal{H}\right\}$. Let $W=P_{\mathcal{K}_{2}}$ be the projection of $\mathcal{K}$ onto $\mathcal{K}_{2}$. Then we see that $V_{1}^{*} V_{2}=V_{1}^{*} W V_{2}$ and $W \pi(a)=\pi(a) W$, so that $\mathcal{J}_{\pi}\left(\phi_{1}, \phi_{2}\right) \subseteq \mathcal{I}\left(\phi_{1}, \phi_{2}\right)$.

Remark 2.6. From the proof of Proposition 2.5, we have that

$$
\mathcal{J}_{\pi}\left(\phi_{1}, \phi_{2}\right) \subseteq \mathcal{I}\left(\phi_{1}, \phi_{2}\right) \subseteq \mathcal{J}_{\widehat{\pi_{1}} \oplus \widehat{\pi_{2}}}\left(\phi_{1}, \phi_{2}\right)
$$

where $\pi$ is a common $J$-representation and $\widehat{\pi}_{i}$ is a minimal $\widehat{J}_{i}$-representation. Hence, we have that

$$
\beta_{\widehat{\pi_{1}} \oplus \widehat{\pi_{2}}}\left(\phi_{1}, \phi_{2}\right) \leq \beta_{\pi}\left(\phi_{1}, \phi_{2}\right)
$$

which implies that the Bures distance between $\alpha$-CP maps is evaluated in the direct sum of the minimal representations.

\section{Estimating Bures Distance}

We recall that the map $x \mapsto \operatorname{Tr}(x(\cdot))$ defines an isometric isomorphism from $\mathcal{B}(\mathcal{H})$ to normalized trace class operators $\mathcal{B}(\mathcal{H})_{*, 1}$.

Theorem 3.1. If $\phi_{i}: \mathcal{A} \rightarrow \mathcal{B}(\mathcal{H})(i=1,2)$ is an $\alpha$-CP map, then we have

$$
=\inf _{V_{1}^{*} V_{2} \in \mathcal{J}\left(\phi_{1}, \phi_{2}\right)}^{\beta\left(\phi_{1}, \phi_{2}\right)^{2}}\left\{\sup _{\rho \in \mathcal{B}(\mathcal{H})_{*, 1}}\left\{\operatorname{Tr}\left(\phi_{1}\left(1_{\mathcal{A}}\right) \rho\right)+\operatorname{Tr}\left(\phi_{2}\left(1_{\mathcal{A}}\right) \rho\right)-2 \operatorname{Re}\left[\operatorname{Tr}\left(V_{1}^{*} V_{2} \rho\right)\right]\right\}\right\} .
$$


Proof. Let $\left(\mathcal{K}, J, \pi, V_{i}\right)$ be a Krein quadruple associated with $\phi_{i}$ in $\alpha-\operatorname{CP}(\mathcal{A}, \mathcal{H})$ $(i=1,2)$. We have that

$$
\begin{aligned}
\left\|V_{1}-V_{2}\right\|^{2} & =\sup _{\rho \in \mathcal{B}(\mathcal{H})_{*, 1}} \operatorname{Tr}\left(\left(V_{1}-V_{2}\right)^{*}\left(V_{1}-V_{2}\right) \rho\right) \\
& =\sup _{\rho \in \mathcal{B}(\mathcal{H})_{*, 1}} \operatorname{Tr}\left(V_{1}^{*} V_{1} \rho+V_{2}^{*} V_{2} \rho-V_{2}^{*} V_{1} \rho-V_{1}^{*} V_{2} \rho\right) \\
& =\sup _{\rho \in \mathcal{B}(\mathcal{H})_{*, 1}}\left\{\operatorname{Tr}\left(\phi_{1}\left(1_{\mathcal{A}}\right) \rho\right)+\operatorname{Tr}\left(\phi_{2}\left(1_{\mathcal{A}}\right) \rho\right)-2 \operatorname{Re}\left[\operatorname{Tr}\left(V_{1}^{*} V_{2} \rho\right)\right]\right\} .
\end{aligned}
$$

By definition of the Bures distance, we obtain that

$$
\begin{aligned}
& \beta\left(\phi_{1}, \phi_{2}\right)^{2} \\
= & \inf _{\pi} \beta_{\pi}\left(\phi_{1}, \phi_{2}\right)^{2}=\inf _{\pi}\left\{\inf _{V_{i} \in S\left(\phi_{i}, \pi\right)}\left\|V_{1}-V_{2}\right\|^{2}\right\} \\
= & \inf _{\pi}\left\{\inf _{V_{i} \in S\left(\phi_{i}, \pi\right)} \sup _{\rho \in \mathcal{B}(\mathcal{H})_{*, 1}}\left\{\operatorname{Tr}\left(\phi_{1}\left(1_{\mathcal{A}}\right) \rho\right)+\operatorname{Tr}\left(\phi_{2}\left(1_{\mathcal{A}}\right) \rho\right)-2 \operatorname{Re}\left[\operatorname{Tr}\left(V_{1}^{*} V_{2} \rho\right)\right]\right\}\right\} \\
= & \inf _{V_{1}^{*} V_{2} \in \mathcal{J}\left(\phi_{1}, \phi_{2}\right)}\left\{\sup _{\rho \in \mathcal{B}(\mathcal{H})_{*, 1}}\left\{\operatorname{Tr}\left(\phi_{1}\left(1_{\mathcal{A}}\right) \rho\right)+\operatorname{Tr}\left(\phi_{2}\left(1_{\mathcal{A}}\right) \rho\right)-2 \operatorname{Re}\left[\operatorname{Tr}\left(V_{1}^{*} V_{2} \rho\right)\right]\right\}\right\} .
\end{aligned}
$$

Remark 3.2. Let $\phi_{i}(i=1,2)$ be $\alpha$-CP maps from $\mathcal{A}$ into $\mathcal{B}(\mathcal{H})$ and let $\left(\widehat{\mathcal{K}}_{i}, \widehat{J}_{i}, \widehat{\pi}_{i}\right.$, $\left.\widehat{V}_{i}\right)$ be a minimal Krein quadruple associated with $\phi_{i}$. Assume that $\left(\mathcal{K}, J, \pi, V_{i}\right)$ is any Krein quadruple associated with $\phi_{i}$. It follows from the proof of Proposition 2.5 that

$$
\mathcal{J}_{\pi}\left(\phi_{1}, \phi_{2}\right) \subseteq \mathcal{I}\left(\phi_{1}, \phi_{2}\right) \subseteq \mathcal{J}_{\widehat{\pi_{1} \oplus \widehat{\pi_{2}}}}\left(\phi_{1}, \phi_{2}\right),
$$

so that $\beta_{\widehat{\pi_{1}} \widehat{\pi_{2}}}\left(\phi_{1}, \phi_{2}\right) \leq \beta_{\pi}\left(\phi_{1}, \phi_{2}\right)$. Hence, we have that $\beta\left(\phi_{1}, \phi_{2}\right)=\beta_{\widehat{\pi_{1}} \oplus \widehat{\pi_{2}}}\left(\phi_{1}, \phi_{2}\right)$. This implies that the Bures distance $\beta\left(\phi_{1}, \phi_{2}\right)$ can be evaluated in the direct sum of the minimal $\widehat{J}_{i}$-representations.

Let $\phi_{i}: \mathcal{A} \rightarrow \mathcal{B}(\mathcal{H})(i=1,2)$ be $\alpha$-CP maps with Krein space dilations $\left(\mathcal{K}_{i}, J_{i}, \pi_{i}, V_{i}\right)$. Then we have that

$$
\begin{aligned}
& \beta\left(\phi_{1}, \phi_{2}\right)^{2} \\
= & \inf _{V_{1}^{*} V_{2} \in \mathcal{J}\left(\phi_{1}, \phi_{2}\right)}\left\{\sup _{\rho \in \mathcal{B}(\mathcal{H})_{*, 1}}\left\{\operatorname{Tr}\left(\phi_{1}\left(1_{\mathcal{A}}\right) \rho\right)+\operatorname{Tr}\left(\phi_{2}\left(1_{\mathcal{A}}\right) \rho\right)-2 \operatorname{Re}\left[\operatorname{Tr}\left(V_{1}^{*} V_{2} \rho\right)\right]\right\}\right\} \\
= & \inf _{V_{1}^{*} W V_{2} \in \mathcal{I}\left(\phi_{1}, \phi_{2}\right)}\left\{\sup _{\rho \in \mathcal{B}(\mathcal{H})_{*, 1}}\left\{\operatorname{Tr}\left(\phi_{1}\left(1_{\mathcal{A}}\right) \rho\right)+\operatorname{Tr}\left(\phi_{2}\left(1_{\mathcal{A}}\right) \rho\right)-2 \operatorname{Re}\left[\operatorname{Tr}\left(V_{1}^{*} W V_{2} \rho\right)\right]\right\}\right\} \\
= & \inf _{\substack{\|W\| \leq 1 \\
W J_{2}=J_{1} W \\
W \pi_{2}(\cdot)=\pi_{1}(\cdot) W}}\left\{\sup _{\rho \in \mathcal{B}(\mathcal{H})_{*, 1}}\left\{\operatorname{Tr}\left(\phi_{1}\left(1_{\mathcal{A}}\right) \rho\right)+\operatorname{Tr}\left(\phi_{2}\left(1_{\mathcal{A}}\right) \rho\right)-2 \operatorname{Re}\left[\operatorname{Tr}\left(V_{1}^{*} W V_{2} \rho\right)\right]\right\}\right\} .
\end{aligned}
$$


Since $\rho \in \mathcal{B}(\mathcal{H})_{*, 1}$ is of trace-class, the operator $V_{2} \rho V_{1}^{*}$ is also of trace-class. The functional $\operatorname{Tr}\left(V_{2} \rho V_{1}^{*}(\cdot)\right)$ is weakly continuous in $\mathcal{W}=\left\{W:\|W\| \leq 1, W J_{2}=\right.$ $\left.J_{1} W, W \pi_{2}(\cdot)=\pi_{1}(\cdot) W\right\}$, so that the infimum is attained by compactness of the set $\mathcal{W}$.

By convexity of the set $\mathcal{W}$, the order of inf and sup can be interchanged in the equation. Hence, we obtain that

$$
\begin{aligned}
& \beta\left(\phi_{1}, \phi_{2}\right)^{2}
\end{aligned}
$$

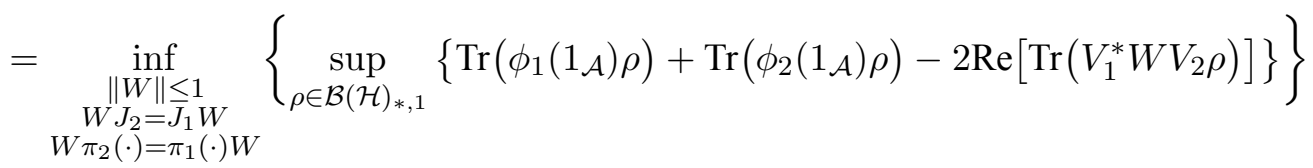

$$
\begin{aligned}
& =\sup _{\rho \in \mathcal{B}(\mathcal{H})_{*, 1}}\left\{\inf _{\substack{\|W\| \leq 1 \\
W J_{2}=J_{1} W \\
W \pi_{2}(\cdot)=\pi_{1}(\cdot) W}}\left\{\operatorname{Tr}\left(\phi_{1}\left(1_{\mathcal{A}}\right) \rho\right)+\operatorname{Tr}\left(\phi_{2}\left(1_{\mathcal{A}}\right) \rho\right)-2 \operatorname{Re}\left[\operatorname{Tr}\left(V_{1}^{*} W V_{2} \rho\right)\right]\right\}\right\} \\
& =\sup _{\substack{\xi \in \mathcal{H} \otimes \mathcal{H} \\
\|\xi\| \leq 1}}\left\{\operatorname { i n f } _ { \substack { \| W \| \leq 1 \\
W J _ { 2 } = J _ { 1 } W \\
W \pi _ { 2 } ( \cdot ) = \pi _ { 1 } ( \cdot ) W } } \left\{\left\langle\xi,\left(\phi_{1}\left(1_{\mathcal{A}}\right) \otimes 1_{\mathcal{H}}\right) \xi\right\rangle+\left\langle\xi,\left(\phi_{2}\left(1_{\mathcal{A}}\right) \otimes 1_{\mathcal{H}}\right) \xi\right\rangle\right.\right. \\
& \left.\left.-2 \operatorname{Re}\left[\left\langle\xi,\left(V_{1}^{*} \otimes 1_{\mathcal{H}}\right)\left(W \otimes 1_{\mathcal{H}}\right)\left(V_{2} \otimes 1_{\mathcal{H}}\right) \xi\right\rangle\right]\right\}\right\} .
\end{aligned}
$$

We denote by $\omega_{\xi, \xi}$ the vector functional by $\xi$ and take $a \in \mathcal{A}$ and $x \in \mathcal{B}(\mathcal{H})$. Then we have that

$$
\left[\omega_{\xi, \xi} \circ\left(\phi_{i} \otimes 1_{\mathcal{B}(\mathcal{H})}\right)\right](a \otimes x)=\left\langle\xi,\left(V_{i}^{*} \otimes 1_{\mathcal{H}}\right)\left(\pi_{i}(a) \otimes x\right)\left(V_{i} \otimes 1_{\mathcal{H}}\right) \xi\right\rangle .
$$

Let $\widetilde{W}=W \otimes 1_{\mathcal{B}(\mathcal{H})}$ be an operator from $\mathcal{K}_{2} \otimes \mathcal{H}$ into $\mathcal{K}_{1} \otimes \mathcal{H}$ where $W: \mathcal{K}_{2} \rightarrow \mathcal{K}_{1}$ is such that $W J_{2}=J_{1} W$ and $W \pi_{2}(a)=\pi_{1}(a) W$ for all $a \in \mathcal{A}$. We immediately see that

$$
\widetilde{W} \widetilde{J}_{2}=\widetilde{J}_{1} \widetilde{W} \quad \text { and } \quad \widetilde{W}\left(\pi_{2}(a) \otimes 1_{\mathcal{B}(\mathcal{H})}\right)=\left(\pi_{1}(a) \otimes 1_{\mathcal{B}(\mathcal{H})}\right) \widetilde{W}, \quad(a \in \mathcal{A})
$$

where $\widetilde{J}_{i}=J_{i} \otimes 1_{\mathcal{H}}$.

Theorem 3.3. Let $\phi_{i}(i=1,2,3)$ be $\alpha$-CP maps from $\mathcal{A}$ into $\mathcal{B}(\mathcal{H})$. The triangle inequality holds; $\beta\left(\phi_{1}, \phi_{3}\right) \leq \beta\left(\phi_{1}, \phi_{2}\right)+\beta\left(\phi_{2}, \phi_{3}\right)$.

Proof. Let $(\mathcal{K}, J, \pi)$ be a common representation for $\phi_{1}$ and $\phi_{2}$, i.e., for $i=1,2$

$$
\phi_{i}(a)=V_{i}^{*} \pi(a) V_{i} \quad \text { and } \quad V_{i}^{*} \pi(a)^{*} \pi(b) V_{i}=V_{i}^{*} \pi\left(\alpha(a)^{*} b\right) V_{i}
$$

where $a, b \in \mathcal{A}$ and let $(\widetilde{\mathcal{K}}, \widetilde{J}, \widetilde{\pi})$ be a common representation for $\phi_{2}$ and $\phi_{3}$ with $\widetilde{V_{1}}$ and $\widetilde{V_{2}}$, respectively. We denote by $\left(\widehat{\mathcal{K}}_{i}, \widehat{J}_{i}, \widehat{\pi}_{i}, \widehat{V}_{i}\right)$ the minimal Krein quadruple 
associated with $\phi_{i}(i=1,2,3)$. Let $U_{i}: \widehat{\mathcal{K}_{i}} \rightarrow \mathcal{K}(i=1,2)$ and $\widetilde{U_{j}}: \widehat{\mathcal{K}_{j}} \rightarrow \widetilde{\mathcal{K}}$ $(j=2,3)$ be isometries satisfying equations $(2.3)$.

We define three maps $\overline{V_{i}}: \mathcal{H} \rightarrow \widehat{\mathcal{K}_{1}} \oplus \widehat{\mathcal{K}_{2}} \oplus \widehat{\mathcal{K}_{3}}(i=1,2,3)$ by

$$
\begin{aligned}
& \overline{V_{1}}=\left(1_{\widehat{\mathcal{K}_{1}}}-U_{1}^{*} U_{2} U_{2}^{*} U_{1}\right)^{\frac{1}{2}} \widehat{V_{1}} \oplus U_{2}^{*} V_{1} \oplus 0, \\
& \overline{V_{2}}=0 \oplus \widehat{V_{2}} \oplus 0, \\
& \overline{V_{3}}=0 \oplus{\widetilde{U_{2}}}^{*} \widetilde{V_{3}} \oplus\left(1_{\widehat{\mathcal{K}_{3}}}-{\widetilde{U_{3}}}^{*} \widetilde{U_{2}} \widetilde{U}_{2}^{*} \widetilde{U_{3}}\right)^{\frac{1}{2}} \widehat{V_{3}} .
\end{aligned}
$$

By letting $\bar{J}:=\widehat{J_{1}} \oplus \widehat{J_{2}} \oplus \widehat{J_{3}}$, we see that $\bar{\pi}:=\widehat{\pi_{1}} \oplus \widehat{\pi_{2}} \oplus \widehat{\pi_{3}}$ is a $\bar{J}$-representation of $\mathcal{A}$ on $\overline{\mathcal{K}}:=\widehat{\mathcal{K}_{1}} \oplus \widehat{\mathcal{K}_{2}} \oplus \widehat{\mathcal{K}_{3}}$, i.e., for all $a, b \in \mathcal{A}$,

$$
\bar{\pi}(a b)=\bar{\pi}(a) \bar{\pi}(b), \quad \bar{\pi}\left(a^{*}\right)=\bar{\pi}(a)^{\bar{J}}
$$

where $\bar{\pi}(a)^{\bar{J}}:=\widehat{\pi_{1}}(a)^{\widehat{J_{1}}} \oplus \widehat{\pi_{2}}(a)^{\widehat{J_{2}}} \oplus \widehat{\pi_{3}}(a)^{\widehat{J_{3}}}$. It immediately follows from definition that $\overline{V_{2}} \in S\left(\phi_{2}, \bar{\pi}\right)$, i.e., for all $a, b \in \mathcal{A}$,

$$
\phi_{2}(a)={\overline{V_{2}}}^{*} \bar{\pi}(a) \overline{V_{2}} \text { and }{\overline{V_{2}}}^{*} \bar{\pi}(a)^{*} \bar{\pi}(b) \overline{V_{2}}={\overline{V_{2}}}^{*} \bar{\pi}\left(\alpha(a)^{*} b\right) \overline{V_{2}} .
$$

For any $a, b \in \mathcal{A}$, we have that

$$
\begin{aligned}
& {\overline{V_{1}}}^{*} \bar{\pi}(a) \overline{V_{1}} \\
= & {\widehat{V_{1}}}^{*}\left(1_{\widehat{\mathcal{K}_{1}}}-U_{1}^{*} U_{2} U_{2}^{*} U_{1}\right)^{\frac{1}{2}} \widehat{\pi_{1}}(a)\left(1_{\widehat{\mathcal{K}_{1}}}-U_{1}^{*} U_{2} U_{2}^{*} U_{1}\right)^{\frac{1}{2}} \widehat{V_{1}}+V_{1}^{*} U_{2} \widehat{\pi_{2}}(a) U_{2}^{*} V_{1} \\
= & V_{1}^{*} U_{1} U_{1}^{*} \pi(a)\left(1_{\widehat{\mathcal{K}_{1}}}-U_{2} U_{2}^{*}\right) U_{1} U_{1}^{*} V_{1}+V_{1}^{*} \widehat{\pi_{1}}(a) U_{2} U_{2}^{*} V_{1} \\
= & \phi_{1}(a)
\end{aligned}
$$

and that

$$
\begin{aligned}
{\overline{V_{1}}}^{*} \bar{\pi}(a)^{*} \bar{\pi}(b) \overline{V_{1}} & =V_{1}^{*} U_{1} U_{1}^{*} \pi(a)^{*} \pi(b)\left(1_{\widehat{\mathcal{K}}_{1}}-U_{2} U_{2}^{*}\right) U_{1} U_{1}^{*} V_{1}+V_{1}^{*} \pi(a)^{*} \pi(b) U_{2} U_{2}^{*} V_{1} \\
& =\phi_{1}\left(\alpha(a)^{*} b\right)={\overline{V_{1}}}^{*} \bar{\pi}\left(\alpha(a)^{*} b\right) \overline{V_{1}} .
\end{aligned}
$$

Hence, $\overline{V_{1}} \in S\left(\phi_{1}, \bar{\pi}\right)$. We similarly get $\overline{V_{3}} \in S\left(\phi_{3}, \bar{\pi}\right)$. Therefore, $(\overline{\mathcal{K}}, \bar{J}, \bar{\pi})$ is a common Krein space representation for $\phi_{1}, \phi_{2}$ and $\phi_{3}$.

Moreover, we have that

$$
\begin{aligned}
& {\overline{V_{2}}}^{*} \overline{V_{1}}=\left(0 \oplus{\widehat{V_{2}}}^{*} \oplus 0\right)\left(\left(1_{\widehat{\mathcal{K}_{1}}}-U_{1}^{*} U_{2} U_{2}^{*} U_{1}\right)^{\frac{1}{2}} \widehat{V_{1}} \oplus U_{2}^{*} V_{1} \oplus 0\right)={\widehat{V_{2}}}^{*} U_{2}^{*} V_{1}=V_{2}^{*} V_{1}, \\
& {\overline{V_{2}}}^{*} \overline{V_{3}}=\left(0 \oplus{\widehat{V_{2}}}^{*} \oplus 0\right)\left(0 \oplus \widetilde{U_{2}}{ }^{*} \widetilde{V}_{3} \oplus\left(1_{\widehat{\mathcal{K}}_{3}}-{\widetilde{U_{3}}}^{*} \widetilde{U_{2}}{\widetilde{U_{2}}}^{*} \widetilde{U_{3}}\right)^{\frac{1}{2}} \widehat{V_{3}}\right)={\widehat{V_{2}}}^{*}{\widetilde{U_{2}}}^{*} \widetilde{V_{3}}={\widetilde{V_{2}}}^{*} \widetilde{V}_{3} .
\end{aligned}
$$


Suppose that $(\mathcal{K}, J, \pi)$ and $(\widetilde{\mathcal{K}}, \widetilde{J}, \widetilde{\pi})$ are chosen as in Remark 3.2, i.e.,

$$
\beta\left(\phi_{1}, \phi_{2}\right)=\beta_{\pi}\left(\phi_{1}, \phi_{2}\right) \text { and } \beta\left(\phi_{2}, \phi_{3}\right)=\beta_{\widetilde{\pi}}\left(\phi_{2}, \phi_{3}\right) .
$$

Thus, we have that

$$
\left\|V_{1}-V_{2}\right\|=\beta_{\pi}\left(\phi_{1}, \phi_{2}\right)=\beta\left(\phi_{1}, \phi_{2}\right) \text { and }\left\|\widetilde{V_{2}}-\widetilde{V_{3}}\right\|=\beta_{\widetilde{\pi}}\left(\phi_{2}, \phi_{3}\right)=\beta\left(\phi_{2}, \phi_{3}\right) .
$$

By the triangle inequality of operator norms, we obtain that

$$
\beta\left(\phi_{1}, \phi_{3}\right) \leq\left\|\overline{V_{1}}-\overline{V_{3}}\right\| \leq\left\|V_{1}-V_{2}\right\|+\left\|\widetilde{V_{2}}-\widetilde{V_{3}}\right\|=\beta\left(\phi_{1}, \phi_{2}\right)+\beta\left(\phi_{2}, \phi_{3}\right),
$$

which completes the proof.

Proposition 3.4. If $\phi_{i}: \mathcal{A} \rightarrow \mathcal{B}(\mathcal{H})(i=1,2)$ are $\alpha$-CP maps with Krein space dilations $\left(\mathcal{K}_{i}, J_{i}, \pi_{i}, V_{i}\right)$, then we have that for any $0 \leq t \leq 1$,

$$
\left|\beta\left(\phi_{1}, \phi_{2}\right)-\beta\left(\phi_{1}, t \phi_{1}+(1-t) \phi_{2}\right)\right| \leq t^{1 / 2}\left(\left\|V_{1}\right\|+\left\|V_{2}\right\|\right) .
$$

Proof. Putting $\widetilde{V_{1}}=V_{1} \oplus 0$ and $\pi=\pi_{1} \oplus \pi_{2}$, we clearly have that $\widetilde{V_{1}} \in$ $S\left(\phi_{1} \oplus \phi_{2}, \pi_{1} \oplus \pi_{2}\right)$. We also see that $\widetilde{V_{2}}=V_{1} \oplus V_{2} \in S\left(\phi_{1} \oplus \phi_{2}, \pi_{1} \oplus \pi_{2}\right)$. Indeed, for any $a, b \in \mathcal{A}$ we have that

$$
\left(\phi_{1} \oplus \phi_{2}\right)(a)=\left(V_{1} \oplus V_{2}\right)^{*}\left(\pi_{1} \oplus \pi_{2}\right)(a)\left(V_{1} \oplus V_{2}\right)=\widetilde{V}_{2}^{*} \pi(a) \widetilde{V}_{2}
$$

and that

$$
{\widetilde{V_{2}}}^{*} \pi(a)^{*} \pi(b) \widetilde{V_{2}}=V_{1}^{*} \pi_{1}\left(\alpha(a)^{*} b\right) V_{1} \oplus V_{2}^{*} \pi_{2}\left(\alpha(a)^{*} b\right) V_{2}={\widetilde{V_{2}}}^{*} \pi\left(\alpha(a)^{*} b\right) \widetilde{V_{2}} .
$$

Moreover, we also see that $\widetilde{V_{1}} \in S\left(\phi_{1} \oplus \phi_{2}, \pi_{1} \oplus \pi_{2}\right)$. Hence, we have that

$$
\beta\left(\phi_{1}, \phi_{1}+\phi_{2}\right) \leq\left\|\widetilde{V_{1}}-\widetilde{V_{2}}\right\|=\left\|V_{2}\right\| .
$$

Let $0 \leq t \leq 1$. By the triangle inequality of the Bures distance, we have that

$$
\begin{aligned}
& \left|\beta\left(\phi_{1}, \phi_{2}\right)-\beta\left(\phi_{1}, t \phi_{1}+(1-t) \phi_{2}\right)\right| \\
\leq & \beta\left(\phi_{2}, t \phi_{1}+(1-t) \phi_{2}\right) \\
\leq & \beta\left(\phi_{2},(1-t) \phi_{2}\right)+\beta\left((1-t) \phi_{2}, t \phi_{1}+(1-t) \phi_{2}\right) \\
\leq & t^{1 / 2}\left(\left\|V_{1}\right\|+\left\|V_{2}\right\|\right),
\end{aligned}
$$

which completes the proof. 


\section{Unbounded Representations of *-Algebras in Krein Spaces}

In this section, we assume that $\mathcal{A}$ is a unital ${ }^{*}$-algebra with unit $1_{\mathcal{A}}$. A representation $\pi$ of $\mathcal{A}$ on a Hilbert space $\mathcal{H}$ is a linear mapping of $\mathcal{A}$ into the algebra of all closable linear operators defined on a common dense subspace $\mathcal{D}(\pi)$ such that

(i) $\mathcal{D}(\pi)$ is globally invariant under $\pi(a)(a \in \mathcal{A})$,

(ii) $\pi\left(1_{\mathcal{A}}\right)=1$, the unit operator,

(iii) $\pi(a) \pi(b) \xi=\pi(a b) \xi$ for all $a, b \in \mathcal{A}, \xi \in \mathcal{D}(\pi)$.

Let $\mathcal{D}\left(\pi^{*}\right):=\cap_{a \in \mathcal{A}} \mathcal{D}\left(\pi(a)^{*}\right)$ and $\pi^{*}(a) \xi:=\pi\left(a^{*}\right)^{*} \xi\left(a \in \mathcal{A}, \xi \in \mathcal{D}\left(\pi^{*}\right)\right)$. Then we see that $\pi^{*}$ is a representation of $\mathcal{A}$ on a Hilbert space $\overline{\mathcal{D}\left(\pi^{*}\right)}$.

If $J$ is a (fundamental) symmetry on $\mathcal{H}$, i.e., $J=J^{*}=J^{-1} \in \mathcal{B}(\mathcal{H})$, then we define a sesquilinear form on $\mathcal{H}$ by

$$
[\xi, \eta]_{J}=\langle J \xi, \eta\rangle .
$$

The pair $(\mathcal{H}, J)$ is called a Krein space. If a representation $\pi$ of $\mathcal{A}$ on $\mathcal{H}$ satisfies

$$
[\pi(a) \xi, \eta]_{J}=\left[\xi, \pi\left(a^{*}\right) \eta\right]_{J}, \quad(a \in \mathcal{A}, \xi, \eta \in \mathcal{H}),
$$

then $\pi$ is called a $J$-representation of $\mathcal{A}$ on $(\mathcal{H}, J)$. Let $T$ be a densely defined linear operator in $(\mathcal{H}, J)$ and let $T^{J}$ be a $J$-adjoint of $T$ defined by

$$
[T \xi, \eta]_{J}=\left[\xi, T^{J} \eta\right]_{J}, \quad\left(\xi \in \mathcal{D}(T), \eta \in \mathcal{D}\left(T^{J}\right) .\right.
$$

We easily see that $T^{J}=J T^{*} J$. We define a $J$-adjoint of $\pi$ by

$$
\mathcal{D}\left(\pi^{J}\right)=\bigcap_{a \in \mathcal{A}} \mathcal{D}\left(\pi(a)^{J}\right), \quad \pi^{J}(a)=\left.\pi\left(a^{*}\right)^{J}\right|_{\mathcal{D}\left(\pi^{J}\right)} .
$$

Then $\pi$ is a $J$-representation of $\mathcal{A}$ if and only if $\mathcal{D}(\pi) \subseteq \mathcal{D}\left(\pi^{J}\right)$ and $\pi(a) \xi=\pi^{J}(a) \xi$ for all $a \in \mathcal{A}$ and $\xi \in \mathcal{D}(\pi)$.

Let $\mathcal{B}$ be a unital ${ }^{*}$-subalgebra of $\mathcal{A}$. Suppose there exists an abstract conditional expectation $P$ of $\mathcal{A}$ onto $\mathcal{B}$ and let $\phi$ be a $P$-functional on $\mathcal{A}$ (see [17] for definition). By [17, Theorem 3], there exists a $J$-representation $\pi$ of $\mathcal{A}$ on a $\operatorname{Krein}$ space $(\mathcal{K}, J)$ with a cyclic vector $\xi$ in $\mathcal{D}(\pi)$. If we define $\alpha$ by

$$
\alpha(a)=2 P(a)-a \text { for all } a \in \mathcal{A},
$$

then $\phi$ is an $\alpha$-completely positive linear functional.

Let $J$-Rep $(\mathcal{A})$ be the set of $J$-representations of $\mathcal{A}$ and let $P$-Ftnal $(\mathcal{A})$ be the set of $P$-functionals on $\mathcal{A}$. For $\phi \in P$-Ftnal $(\mathcal{A})$ and $\pi \in J-\operatorname{Rep}(\mathcal{A})$, we denote by $S(\phi, \pi)$ the set of unit vectors $\xi \in \mathcal{D}(\pi)$ such that $\phi(a)=\langle\pi(a) \xi, \xi\rangle$. For any $P$-functionals $\phi_{1}$ and $\phi_{2}$ in $P$-Ftnal $(\mathcal{A})$, let $\mathfrak{F}\left(\phi_{1}, \phi_{2}\right)$ be the set of all linear functionals $F$ on $\mathcal{A}$ such that 
(1) $F(\alpha(a) \alpha(b))=F(\alpha(a b))$,

(2) $\left|F\left(\alpha\left(a^{*}\right) b\right)\right|^{2} \leq \phi_{2}\left(\alpha\left(a^{*}\right) a\right) \phi_{1}\left(\alpha\left(b^{*}\right) b\right)$,

where $\alpha$ is a bounded Hermitian map on $A$ with $\alpha^{2}=\mathrm{id}_{\mathcal{A}}$. We call any linear functional in $\mathfrak{F}\left(\phi_{1}, \phi_{2}\right)$ a transition form from $\phi_{1}$ to $\phi_{2}$.

Definition 4.1. Let $\phi_{i}(i=1,2)$ be any elements of $P$-Ftnal $(\mathcal{A})$. We define the transition probability between $\phi_{1}$ and $\phi_{2}$ by

$$
\mathcal{P}\left(\phi_{1}, \phi_{2}\right)=\sup _{\pi \in J-\operatorname{Rep}(\mathcal{A})} \sup _{\xi_{i} \in S\left(\phi_{i}, \pi\right)}\left|\left\langle\xi_{1}, \xi_{2}\right\rangle\right|^{2} .
$$

In the case of von Neumann algebras and normal states, the definition of the transition probability appears in [6]. In general case of *-algebras and states, the definition and explicit formulae were given by Uhlmann [19]. The Bures distance between $P$-functionals $\phi_{1}$ and $\phi_{2}$ is given by $\beta\left(\phi_{1}, \phi_{2}\right)=\inf \left\{\left\|\xi_{1}-\xi_{2}\right\|: \xi_{i} \in\right.$ $\left.S\left(\phi_{i}, \pi\right), \pi \in J-\operatorname{Rep}(\mathcal{A})\right\}$. As in the case of normal states, the Bures distance between $P$-functionals is also related to the transition probability by the formula $\beta\left(\phi_{1}, \phi_{2}\right)^{2}=$ $2\left(1-\mathcal{P}\left(\phi_{1}, \phi_{2}\right)^{1 / 2}\right)$.

For any $\xi_{1} \in S\left(\phi_{1}, \pi\right)$ and $\xi_{2} \in S\left(\phi_{2}, \pi\right)$, we define a functional $F_{\xi_{1}, \xi_{2}}$ on $\mathcal{A}$

$$
F_{\xi_{1}, \xi_{2}}(a)=\left\langle\pi(a) \xi_{1}, \xi_{2}\right\rangle .
$$

Let $a, b \in \mathcal{A}$. Then we have that

$$
\begin{aligned}
F_{\xi_{1}, \xi_{2}}(\alpha(a b)) & =\left\langle\pi(\alpha(a b)) \xi_{1}, \xi_{2}\right\rangle=\overline{\left\langle\pi\left(b^{*}\right) \pi\left(a^{*}\right) \xi_{2}, \xi_{1}\right\rangle} \\
& =\left\langle\pi(\alpha(a)) \pi(\alpha(b)) \xi_{1}, \xi_{2}\right\rangle=F_{\xi_{1}, \xi_{2}}(\alpha(a) \alpha(b))
\end{aligned}
$$

and that

$$
\left|F_{\xi_{1}, \xi_{2}}\left(\alpha\left(a^{*}\right) b\right)\right|^{2} \leq\left\langle\pi\left(\alpha\left(a^{*}\right) a\right) \xi_{2}, \xi_{2}\right\rangle\left\langle\pi\left(\alpha\left(b^{*}\right) b\right) \xi_{1}, \xi_{1}\right\rangle=\phi_{2}\left(\alpha\left(a^{*}\right) a\right) \phi_{1}\left(\alpha\left(b^{*}\right) b\right) .
$$

Hence, we see that $F_{\xi_{1}, \xi_{2}} \in \mathfrak{F}\left(\phi_{1}, \phi_{2}\right)$.

The following theorem was proved by Alberti [2] for states on $C^{*}$-algebras and by Uhlmann [20] for states for *-algebras.

Theorem 4.2. If $\phi_{1}$ and $\phi_{2}$ are P-functional on $\mathcal{A}$, then

$$
\mathcal{P}\left(\phi_{1}, \phi_{2}\right)=\sup _{F \in \mathfrak{F}\left(\phi_{1}, \phi_{2}\right)}\left|F\left(1_{\mathcal{A}}\right)\right|^{2} .
$$

Proof. Assume that $\pi$ is a $J$-representation of $\mathcal{A}$ on a Krein space $(\mathcal{K}, J)$ such that $S\left(\phi_{1}, \pi\right)$ and $S\left(\phi_{2}, \pi\right)$ are non-empty. Take $\xi_{1} \in S\left(\phi_{1}, \pi\right)$ and $\xi_{2} \in S\left(\phi_{2}, \pi\right)$ and we define two Hilbert spaces

$$
\mathcal{K}_{1}=\overline{\left\{\pi(a) \xi_{1}: a \in \mathcal{A}\right\}} \quad \text { and } \quad \mathcal{K}_{2}=\overline{\left\{\pi(a) \xi_{2}: a \in \mathcal{A}\right\}} .
$$


Let $P_{1}: \mathcal{K} \rightarrow \mathcal{K}_{1}$ and $P_{2}: \mathcal{K} \rightarrow \mathcal{K}_{2}$ be orthogonal projections. Then we see that $P_{1}$ and $P_{2}$ belong to the commutant $\pi(\mathcal{A})^{\prime}$.

Let $F \in \mathfrak{F}\left(\phi_{1}, \phi_{2}\right)$. We have that

$$
\left|F\left(\alpha\left(a^{*}\right) b\right)\right|^{2} \leq \phi_{2}\left(\alpha\left(a^{*}\right) a\right) \phi_{1}\left(\alpha\left(b^{*}\right) b\right)=\left\|\pi(a) \xi_{2}\right\|^{2}\left\|\pi(b) \xi_{1}\right\|^{2}
$$

for all $a, b \in \mathcal{A}$, so that the map $\left(\pi(b) \xi_{1}, \pi(a) \xi_{2}\right) \mapsto F\left(\alpha\left(a^{*}\right) b\right)$ is a densely defined bounded sesquilinear form on $\mathcal{K}_{1} \times \mathcal{K}_{2}$. Therefore, there exist a unique bounded operator $T$ from $\mathcal{K}_{1}$ into $\mathcal{K}_{2}$ such that

$$
F\left(\alpha\left(a^{*}\right) b\right)=\left\langle T \pi(b) \xi_{1}, \pi(a) \xi_{2}\right\rangle .
$$

It is obvious that $\|T\| \leq 1$. Putting $K:=P_{2} T P_{1} \in \mathcal{B}(\mathcal{K})$, we have that $\|T\| \leq 1$ and that

$$
\begin{aligned}
\left\langle K \pi(a) \pi(c) \xi_{1}, \pi(c) \xi_{2}\right\rangle & =\left\langle T \pi(a b) \xi_{1}, \pi(b) \xi_{2}\right\rangle \\
& =F\left(\alpha\left(b^{*}\right) a c\right) \\
& =\left\langle T \pi(c) \xi_{1}, \pi\left(\alpha\left(a^{*}\right) b\right) \xi_{2}\right\rangle \\
& =\left\langle\pi(a) K \pi(c) \xi_{1}, \pi(b) \xi_{2}\right\rangle,
\end{aligned}
$$

which implies that $K \in \pi(\mathcal{A})^{\prime}$.

It follows from Theorem of Russo and Dye that for any $\epsilon>0$, there are nonnegative real numbers $r_{1}, \ldots, r_{n}$ with $\sum_{i} r_{i}=1$ and unitaries $U_{1}, \ldots, U_{n} \in \pi(\mathcal{A})^{\prime}$ such that

$$
\left\|K-\sum_{i=1}^{n} r_{i} U_{i}\right\|<\epsilon .
$$

Hence, $\left|F\left(1_{\mathcal{A}}\right)-\sum_{i=1}^{n} r_{i}\left\langle U_{i} \xi_{1}, \xi_{2}\right\rangle\right|<\epsilon$, so that $\left|F\left(1_{\mathcal{A}}\right)\right|<\epsilon+\sum_{i=1}^{n} r_{i}\left|\left\langle U_{i} \xi_{1}, \xi_{2}\right\rangle\right|$. Since $U_{i} \in \pi(\mathcal{A})^{\prime}$ implies $U_{i} \xi_{1} \in S\left(\phi_{2}, \pi\right)$, we have that

$$
\left|F\left(1_{\mathcal{A}}\right)\right|<\epsilon+\mathcal{P}\left(\phi_{1}, \phi_{2}\right)^{1 / 2} \text {. }
$$

Since $\epsilon>0$ was arbitrary, $\left|F\left(1_{\mathcal{A}}\right)\right| \leq \mathcal{P}\left(\phi_{1}, \phi_{2}\right)^{1 / 2}$.

Let $\epsilon>0$ be given. Assume that $\xi_{1} \in S\left(\phi_{1}, \pi\right)$ and $\xi_{2} \in S\left(\phi_{2}, \pi\right)$ are such that

$$
\left|\left\langle\xi_{1}, \xi_{2}\right\rangle\right|^{2} \geq \mathcal{P}\left(\phi_{1}, \phi_{2}\right)-\epsilon .
$$

By defining $F(a)=\left\langle\pi(a) \xi_{1}, \xi_{2}\right\rangle$, we have that $F \in \mathfrak{F}\left(\phi_{1}, \phi_{2}\right)$ and $\left|F\left(1_{\mathcal{A}}\right)\right|^{2} \geq$ $\mathcal{P}\left(\phi_{1}, \phi_{2}\right)-\epsilon$. Since $\epsilon>0$ was arbitrary, we obtain that

$$
\sup _{F \in \mathfrak{F}\left(\phi_{1}, \phi_{2}\right)}\left|F\left(1_{\mathcal{A}}\right)\right|^{2} \geq \mathcal{P}\left(\phi_{1}, \phi_{2}\right),
$$

which completes the proof. 


\section{ACKNOWLEDGMENT}

A part of this research was carried out while the author was visiting the Korean Institute for Advanced Study (KIAS). He is grateful to professors and the staff in the KIAS for their warm hospitality. The author would also like to thank the referee for careful reading of the manuscript and a number of helpful comments.

\section{REFERENCES}

1. S. Abe, Nonadditive generalization of the quantum Kullback-Leibler divergence for measuring the degree of purification, Phys. Rev. A, 68 (2003), 032302.

2. P. Alberti, A note on the transition probability over $C^{*}$-algebras, Lett. Math. Phys., 7 (1983), 25-32.

3. P. Alberti, Playing with fidelities, Rep. Math. Phys., 51 (2002), 87-125.

4. J. P. Antoine and S. Ôta, Unbounded GNS representations of *-algebra in a Krein space, Lett. Math. Phys., 18 (1989), 267-274.

5. B. V. Rajarama Bhat and K. Sumesh, Bures Distance for Completely Positive Maps, arXiv preprint arXiv:1305.0111v1, 2013.

6. D. Bures, An extension of Kakutani's theorem on infinite product measures to the tensor product of semifinite $W^{*}$-algebras, Trans. Amer. Math. Soc., 135 (1969), 199-212.

7. E. B. Davies, Quantum Theory of Open Systems, Academic Press, London, 1976.

8. J. Heo, $\alpha$-Completely positive maps of group systems and Krein module representations, J. Math. Anal. Appl., 409 (2014), 544-555.

9. J. Heo, J. P. Hong and U. C. Ji, On KSGNS representations on Krein $C^{*}$-modules, J. Math. Phys., 51 (2010), 053504.

10. J. Heo and U. C. Ji, Radon-Nikodým type theorem for $\alpha$-completely positive maps, $J$. Math. Phys., 51 (2010), 103505.

11. J. Heo, U. C. Ji and Y. Kim, $\alpha$-Completely positive maps on locally $C^{*}$-algebras, Krein modules and Radon-Nikodým theorem, J. Korean Math. Soc., 50 (2013), 61-80.

12. J. Heo, U. C. Ji and Y. Kim, Projective covariant representation of locally $C^{*}$-dynamical systems, Taiwanese J. Math., 17 (2013), 529-544.

13. J. Heo, U. C. Ji and Y. Kim, Covariant representations on Krein $C^{*}$-modules associated to pairs of two maps, J. Math. Anal. Appl., 398 (2013), 35-45.

14. G. Hofmann, An explicit realization of a GNS representation in a Krein-space, Publ. RIMS, Kyoto Univ., 29 (1993), 267-287.

15. L. Jakóbczyk, Borchers algebra formulation of an indefinite inner product quantum field theory, J. Math. Phys., 25 (1984), 617-622. 
16. D. Kretschmann, D. Schlingemann and R. Werner, A continuity theorem for Stinespring's dilation, J. Funct. Anal., 255 (2008), 1889-1904.

17. S. Ôta, Unbounded GNS representations of a *-algebra in a Krein space, Letters in Math. Phys., 18 (1989), 267-274.

18. F. Strocchi, Spontaneous symmetry breaking in local gauge quantum field theory; the Higgs mechanism, Comm. Math. Phys., 56 (1977), 57-78.

19. A. Uhlmann, The "transition probability" in the state space of a *-algebra, Rep. Mathematical Phys., 9 (1976), 273-279.

20. A. Uhlmann, The transition probability for states of *-algebras, Ann. Phys., 42 (1985), 524-532.

Jaeseong Heo

Department of Mathematics

Research Institute for Natural Sciences

Hanyang University

Seoul 133-791

Korea

E-mail: hjs@hanyang.ac.kr 